\title{
Remote-Field Manifestations of Mental Activity of the Human Brain
}

\author{
Evgeny A. Yumatov, Elena V. Bykova, Olga V. Potapova, Aligeydar A. Ragimov, \\ Emil L. Salimov \\ I. M. Sechenov First Moscow State Medical University, Moscow, Russia \\ Email: eayumatov@mail.ru
}

Received 7 March 2015; accepted 1 May 2015; published 6 May 2015

Copyright (C) 2015 by authors and Scientific Research Publishing Inc.

This work is licensed under the Creative Commons Attribution International License (CC BY). http://creativecommons.org/licenses/by/4.0/

(c) (i) Open Access

\begin{abstract}
The aim of the present study is to investigate mental activity of the brain. The article deals with external remote-field manifestation of the subjective state of a person. There are statistically significant results obtained which demonstrate contactless remote influence of the subjective state of a person on erythrocyte sedimentation rate in different blood groups, Rh-positive and Rh-negative blood, and under different conditions of exposure. The authors set up a hypothesis of the existence of molecular structures in the blood, which can provide a remote response to expressed subjective state of a person.
\end{abstract}

\section{Keywords}

Psyche, Mentality, Consciousness, Brain, Blood, Erythrocyte Sedimentation Rate (ESR)

\section{Introduction}

I. M. Sechenov laid the foundation for the beginning of the objective study of mental activity of the brain, whereby he was the first to suggest the unity of physical and mental processes [1].

As it is known, mental activity of the brain is manifested in consciousness, thoughts, feelings, emotions, i.e., in the subjective person's perception of himself, and the world around him.

That mental activity of the brain needed broad investigation was asserted by Pavlov, Sperry, Crick et al., Anokhin, Bekhtereva, Ivanitskiy, Nagel, Popper and others [2]-[9].

Unfortunately, at the present time some physiologists believe that neurophysiological phenomena themselves represent manifestations of the mental function within the context of the brain activity, while others pretend that such challenge does not exist at all, and make any attempts to explore the mysteries of mental activity of the brain with a pinch of salt. 
Great achievements of modern neurophysiology which are based on structural, morphological, electrophysiological, neurochemical, molecular, and genetic studies, as well as on data of computed tomography, etc., alone do not provide any understanding of the nature of mental activity of the brain. It is impossible to explain how, based on codes of nerve impulses, interactions among various structures of the brain, electrophysiological processes, molecular transformations and so on, the self-awareness of subjective state develops.

According to Nagel, there is a "gap" in understanding of mental and neurophysiological phenomena. "Without changing the basic conception of consciousness, there is no prospect of overcoming the explanatory gap" [8].

The existing gap between the actual knowledge in the field of neurophysiology of the brain and the idea of mental functions of the brain is due to the fact that investigation of the brain has always relied upon morphological, physical, and chemical methods, which are based on data, phenomena, physical and chemical laws, which have been discovered in inanimate nature. To be able to perform objective investigations of the subjective state, fundamentally different scientific methods and approaches are needed.

The subjective state is intrinsic to the living organization only, and does not exist in inanimate nature. Therefore, it is impossible to explain the origin of mental functions of the brain in view of the laws of inanimate nature only.

In living organism and in particular in the brain, such physical phenomena and processes may occur, which in principle, do not exist and cannot exist in inanimate nature. This thesis is of fundamental importance for understanding of the very core of the subjective in the activity of the brain.

In Popper's view, cognition of the subjective activity of the brain is a "psychophysical problem", and to solve this problem, "changes in the fundamental concepts of consciousness" are required [9].

The general purpose of our research is to reveal molecular and psychophysical mechanisms of mental activity of the brain [10] [11].

Our investigations of mental activity of the brain are based on our methodological principle, which states "subjective processes can be directly registered and studied only using and involving living structures" [10]-[12]. Using this principle, we have conducted a series of studies in which the possibility of direct remote registration of the human subjective state is shown for the first time [13]-[15].

To study the remote effects of the expressed subjective state of a person, we used blood as a biological substrate, since it was a universal multi-component liquid containing cellular elements and protein and colloid and electrolyte solutions.

In previous experiments, it has been shown that the expressed subjective state of a person provides remotefield effects of erythrocyte sedimentation rate (ESR) of blood [16], which is determined by complex physical and chemical interactions of electrostatic potentials of red blood cells, as well as protein and colloidal properties of blood plasma.

Our findings indicate the existence of certain molecular factors present in the blood, which responds remotely to mental state of a person. These results have defined a new area of research, which is focused on search and isolation from the blood of specific molecules, which respond remotely to subjective state of a person.

The specific objectives of the study were:

1) To investigate the effects of the expressed subjective state of a person on ESR in different blood groups and in Rh-positive and Rh-negative blood;

2) To compare the effects of remote influence of the expressed subjective state of a person on ESR in blood placed in Panchenkov apparatus (blood sedimentation rack) and in tubes.

\section{Materials, Methods}

In clinical settings, 2 to $5 \mathrm{ml}$ blood with a known blood group and $\mathrm{Rh}$ factor was sampled with addition of EDTA anticoagulant from healthy men, which were between 18 and 35 years old. ESR was determined according to the standard method Panchenkov. Capillaries with blood were placed in separate racks, some of which were control racks and these did not get any subjective exposure; and others were experimental racks, where the blood was previously exposed to the expressed subjective state of the investigator.

In order to evaluate the possible variation (range) of ESR values, in a number of experiments double control was used. For this purpose, in each rack (control and experimental) several capillaries were placed, which were equally filled with blood. At the same time, the sequence of filling and placement of capillaries with blood in control and test racks varied, additionally, two control racks were used. 
The investigator promoted his own expressed subjective state using "imaginary-cogitative method" [12] [13] [15]. When conducting this subjective test, the investigator was maximally focusing and concentrating his attention on something emotionally significant, whereby he induced in himself an expressed emotional stress.

When carrying out experimental tests, the investigator five times one after another, within 30 seconds, approached the experimental rack with blood in his expressed subjective state, during which time the rack with capillaries was placed horizontally and oriented so that the upper end of capillaries was directed towards the investigator. Control racks at this time were located at a distance, in the other room. Change of position of experimental and control racks to a vertical one was performed simultaneously.

In previous experiments, it was found that remote influence of the subjective state of a person on ESR takes place only when the investigator is located no more than $80-100 \mathrm{~cm}$ away from the capillaries with blood [16].

When carrying out the subjective influence on blood, we used previously developed by our working group indicators of the subjective state, which always used to provide to the observers present during the experiment the exact information on the subjective state of the investigator at the moment when he was approaching the rack with blood-filled capillaries [16]. At the same time, the investigator himself could access his own subjective state without any indicators.

All tests were conducted under constant conditions and at the same time. Within each experiment, blood of the same person was used for all tests. ESR readings performed one hour after setting of racks in a vertical position.

The results for ESR readings were compared between control and test blood using data from capillaries with blood from different racks. In total, 31 experiments were carried out, including 135 tests. Statistical significance of the results was determined by Student's test.

There were two sets of experiments.

In the first series, we studied the effects of the direct subjective influence on blood in capillaries of the Panchenkov apparatus.

In the second series of experiments, the effects of subjective influence on blood in tubes were studied. When preparing the test, each blood sample was equally divided into two portions filled into two tubes, one of which was a control tube and the other one an experimental tube. Similar to the first series of experiments, during experimental tests, the investigator approached five consecutive times, for 30 seconds to a tube with blood, being at those moments in the expressed subjective state. At that time, the control tube was located in another room. Thereafter, blood from the experimental and control tubes was filled into capillaries, which separately installed in racks for subsequent determination of ESR as described earlier procedure.

\section{Results}

\subsection{ESR in Blood in Panchenkov Apparatus after Subjective Influence by Investigator}

Table 1 shows ESR values registered in control and experimental tests after expressed subjective influence of the investigator on the blood in capillaries of the experimental rack. It should be noted that variations in ESR values in each of the control and experimental tests were either absent or minor, compared to changes which took place in experimental tests.

The results (Table 1$)$ demonstrate highly significant $(\mathrm{p}<0.0001)$ differences in ESR between experimental and control tests across the entire set of data. In some tests, the values differed 2-fold or even more. It was found, that significant differences in ESR between control and experimental tests were not associated with blood group.

There were significant differences in ESR changes in experimental tests compared with control, which depended on the presence of $\mathrm{Rh}$ factor.

Exposure of Rh-positive blood to subjective influence leads to highly significant $(\mathrm{p}<0.0001)$ decrease in ESR in experimental tests compared to controls (Table 1, Figure 1).

Conversely, when Rhesus-negative blood samples were exposed, highly significant $(\mathrm{p}<0.0001)$ increase in ESR rate was observed in experimental tests compared to control (Table 1, Figure 1).

There was a 100\%-correlation between the Rh factor and the direction of ESR changes in experimental tests compared to control.

These data indicate that regardless of the presence or absence of the Rh factor, there was always the reaction of blood to remote influence, leading to significant changes in ESR in response to expressed subjective state of a person. However, in the presence of the Rh factor, the direction of changes of ESR reverses. It can be suggested, 


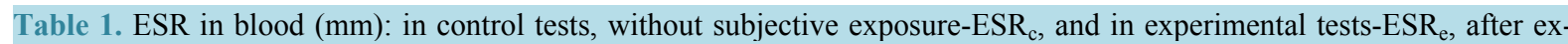
pressed subjective exposure of blood in capillaries, placed in Panchenkov apparatus. Each line represents ESR values in separate tests within the same experiment, blood group and Rh factor presence. Number of experiments-22, number of tests86. $\mathrm{p}$-value is a significance of the difference in ESR level between the control and experimental groups.

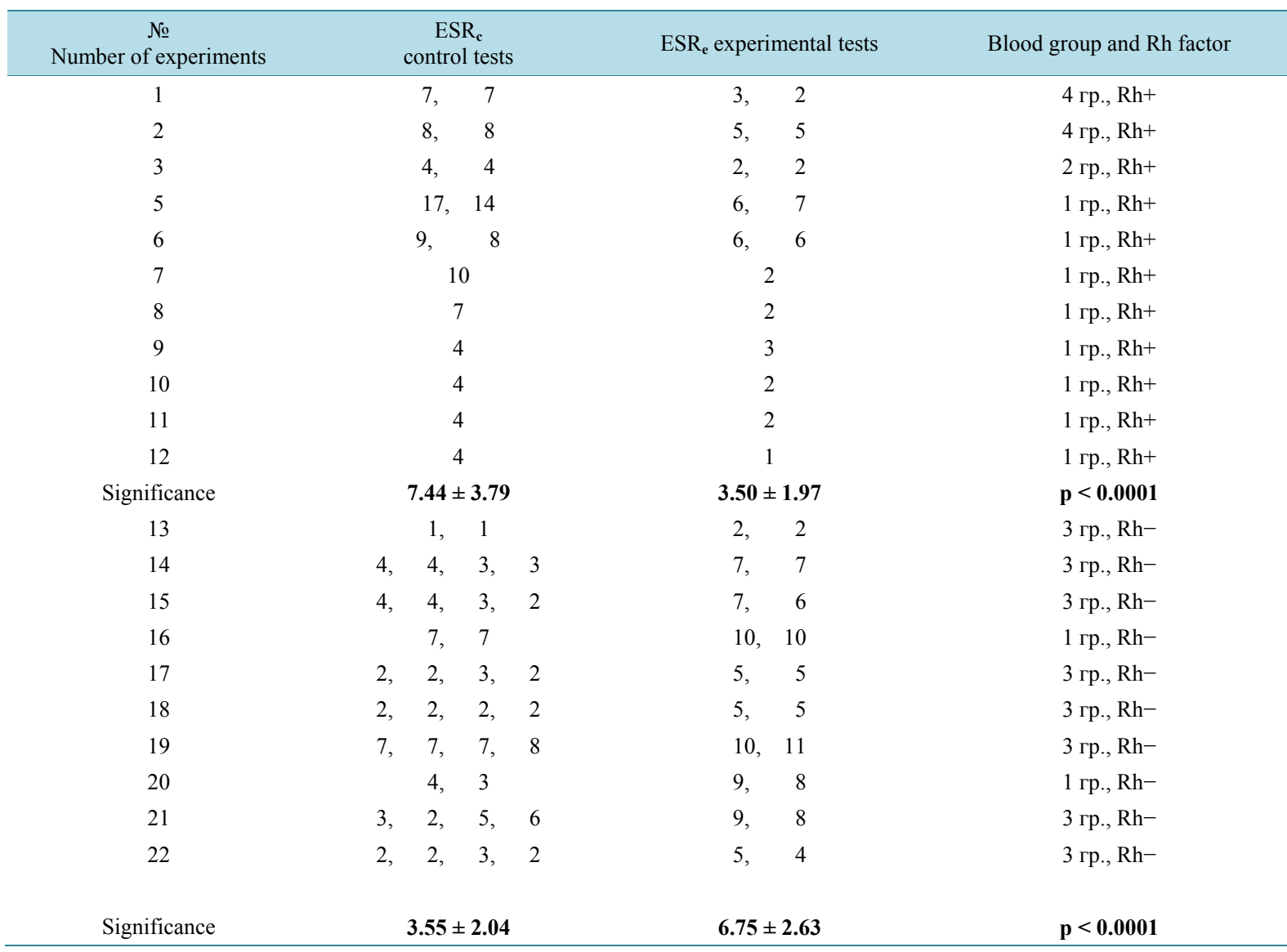

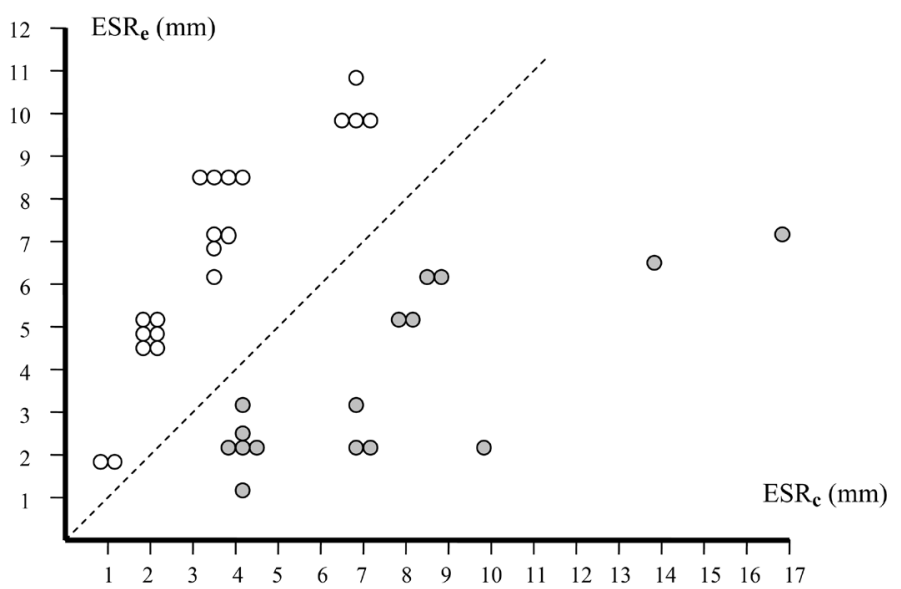

Figure 1. Changes in erythrocyte sedimentation rate (ESR) after exposure of blood in Panchenkov apparatus capillaries to expressed subjective remote influence of the investigator. Legend: $x$-axis: background values $\operatorname{ESR}_{\mathrm{c}}(\mathrm{mm})$ in each individual test in the control; y-axis: experimental values $\operatorname{ESR}_{\mathrm{e}}(\mathrm{mm})$ in each individual test after subjective exposure, relative to the background value in the control-ESR . n-total number of measurements $\mathrm{ESR}_{\mathrm{c}}+\mathrm{ESR}_{\mathrm{e}}=86$. Blood: Rh- O, Rh+O Significance of the difference in ESR in the control and after subjective exposure in $R h+$ blood $p<0.0001$, in Rh-blood $p<$ 0.0001. - - an imaginary line on which experimental values $\mathrm{ESR}_{\mathrm{e}}$ would be located, if they were equal to control values $\mathrm{ESR}_{\mathrm{c}}: \mathrm{ESR}_{\mathrm{c}}=\mathrm{ESR}_{\mathrm{e}}$. Above the line: $\mathrm{ESR}_{\mathrm{e}}>\mathrm{ESR}_{\mathrm{c}}$, below the line: $\mathrm{ESR}_{\mathrm{e}}<\mathrm{ESR}_{\mathrm{c}}$. 
that such effect occurs due to the combined remote-field action of the subjective state of a person on various molecular structures of blood. Apparently, in the absence of the Rh factor, the effect of the subjective state of a person on ESR is shown through other molecular structures of blood.

\subsection{ESR in Blood in a Tube after Subjective Influence by Investigator}

The aim of this series of experiments was to develop a methodology for subsequent identification of molecular structures in the blood, which are remotely responsive to the expressed subjective state of a person.

It is important to know, whether the effect of the remote subjective influence of a person on human blood is maintained after blood mixing?

For example, it is known that in magnetic field iron filings are aligned along field lines. However, after removal of the magnet and shaking iron filings, they do not return to the previous orientation.

In the present study, after exposure of the blood in a tube to the subjective influence and subsequent transfer of this blood into capillaries for ESR determination, blood mixing occurs inevitably and this can affect or eliminate the detected effect of remote influence of the subjective state of a person on ESR for blood in capillaries.

Table 2 shows the results for ESR values registered in control and experimental tests after remote influence of the expressed subjective condition of a person on blood in a test tube. It can be observed that in each control and experimental test variations in ESR were either absent or minor compared to the changes observed in experimental tests.

The results demonstrate (Table 2, Figure 2) highly significant $(p<0.0001 ; p<0.001)$ differences in ESR between experimental and control tests on the entire data set. In contrast to the first series of experiments, in this series the ESR changes in experimental tests in comparison with the control were unidirectional both for Rhpositive and Rh-negative blood. This effect cannot be attributed to the difference in blood groups, as from the results of the first series of experiments we see that the direction of ESR changes does not depend on a blood group.

The main outcome of this series of experiments is the finding that after mixing of blood, the effects of remote subjective human influence on ESR do not disappear.

After mixing, molecular structures present within blood, maintain the changes caused by the previous influence of the subjective state of a person. At the same time, significant differences in ESR between control and experimental tests present in different blood groups.

Table 2. ESR in blood ( $\mathrm{mm}$ ): in control tests, without subjective exposure-ESR $\mathrm{E}_{\mathrm{c}}$, and in experimental tests-ESR $\mathrm{E}_{\mathrm{e}}$, after expressed subjective exposure of blood in a tube. Each line represents ESR values in separate tests within the same experiment, blood group and Rh factor presence. Number of experiments-9, number of tests-49. p-value is a significance of the difference in ESR level between the control and experimental groups.

\begin{tabular}{|c|c|c|c|c|c|c|}
\hline \multirow{2}{*}{$\begin{array}{c}\text { № } \\
\text { Number of experiments } \\
1\end{array}$} & \multicolumn{4}{|c|}{$\begin{array}{c}\mathrm{ESR}_{\mathrm{c}} \\
\text { control tests }\end{array}$} & $\mathrm{ESR}_{\mathrm{e}}$ experimental tests & \multirow{2}{*}{$\begin{array}{c}\text { Blood group and } \mathrm{Rh} \text { factor } \\
1 \text { гр., } \mathrm{Rh}+\end{array}$} \\
\hline & 2 & 2 , & 1 & 1 & 4 & \\
\hline 2 & 3 & 3 , & 2 & 2 & $10, \quad 9$ & 1 гр., Rh+ \\
\hline 3 & 2 & 2 , & 2 & 2 & 5 & 1 гр., $\mathrm{Rh}+$ \\
\hline 4 & \multicolumn{4}{|c|}{$14, \quad 13$} & $17, \quad 16, \quad 17$ & 1 гр., $\mathrm{Rh}+$ \\
\hline 5 & 4 & 4 , & 5 & 5 & 8 & 1 гр., Rh+ \\
\hline 6 & 3 & 3 , & 3 & 3 & 6 & 1 гр., Rh+ \\
\hline Significance & \multicolumn{4}{|c|}{$4.50 \pm 4.34$} & $8.25 \pm 4.31$ & $\mathrm{p}<0.0001$ \\
\hline 7 & 1 , & 1 , & 1 & 1 & 2 & 3 гр., $\mathrm{Rh}^{-}$ \\
\hline 8 & & 1 , & 1 & & 2 & 3 гр., $\mathrm{Rh}^{-}$ \\
\hline 9 & & 1 , & 2 & & 2 & 3 гр., $\mathrm{Rh}^{-}$ \\
\hline Significance & & 1.17 & 0.41 & & $2.33 \pm 0.82$ & p $<0.001$ \\
\hline
\end{tabular}




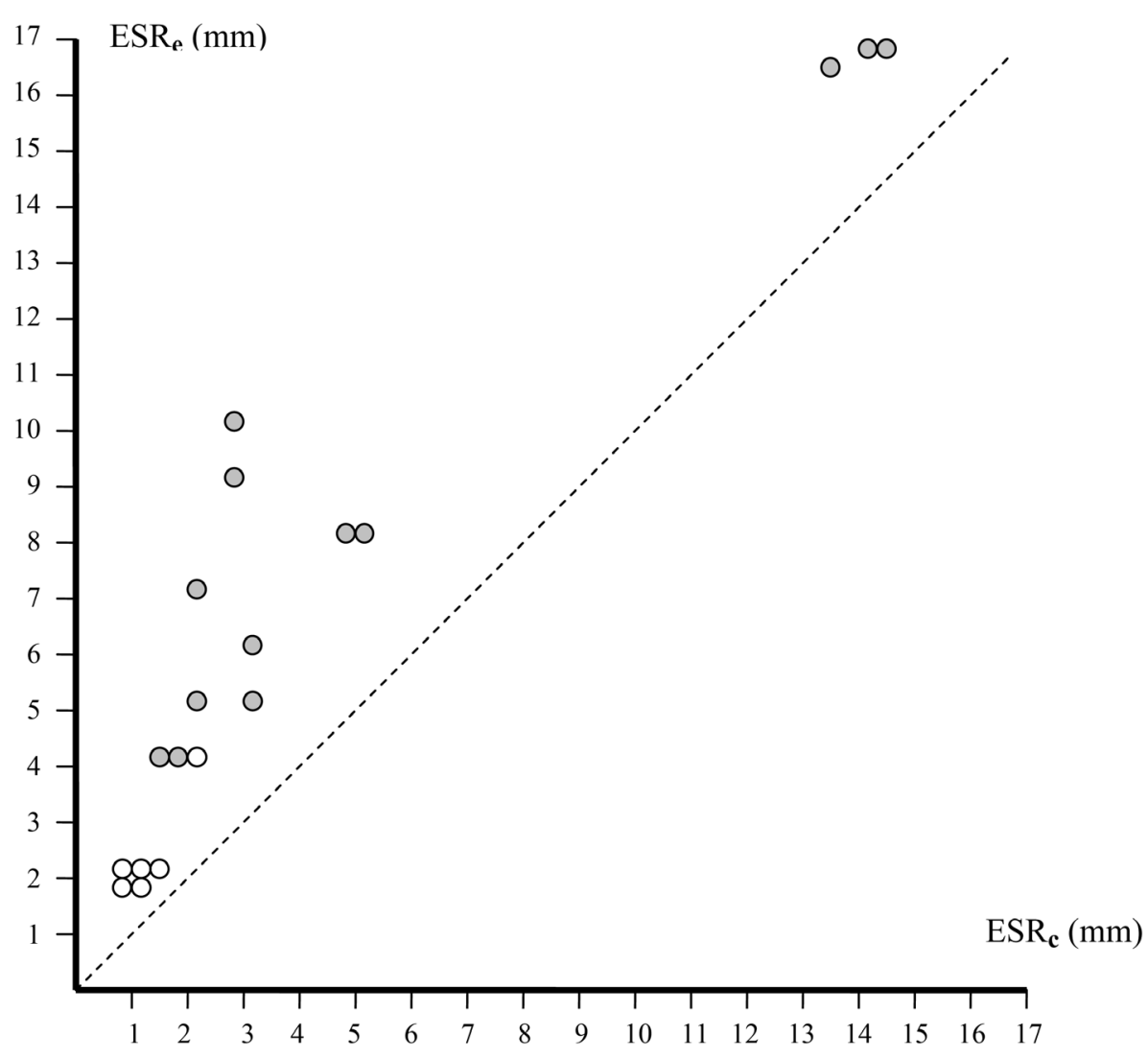

Figure 2. Changes in erythrocyte sedimentation rate (ESR) after exposure of blood in a tube to expressed subjective remote influence of the investigator. Legend: $x$-axis: background values $\operatorname{ESR}_{\mathrm{c}}(\mathrm{mm})$ in each individual test in the control; y-axis: experimental values $\operatorname{ESR}_{\mathrm{e}}(\mathrm{mm})$ in each individual test after subjective exposure, relative to the background value in the control-ESR. n-total number of measurements $\mathrm{ESR}_{\mathrm{c}}+\mathrm{ESR}_{\mathrm{e}}=49$. Blood: $\mathrm{Rh}-\mathrm{O}, \mathrm{Rh}+\mathrm{O}$ Significance of the difference in ESR in the control and after subjective exposure in $\mathrm{Rh}+$ blood $\mathrm{p}<0.0001$, in Rh-blood $\mathrm{p}<0.001$. - - an imaginary line on which experimental values $\mathrm{ESR}_{\mathrm{e}}$ would be located, if they were equal to control values $\mathrm{ESR}_{\mathrm{c}}: \mathrm{ESR}_{\mathrm{c}}=\mathrm{ESR}_{\mathrm{e}}$. Above the line: $\mathrm{ESR}_{\mathrm{e}}>\mathrm{ESR}_{\mathrm{c}}$, below the line: $\mathrm{ESR}_{\mathrm{e}}<\mathrm{ESR}_{\mathrm{c}}$.

These findings provide a reference point for further research. Apparently, significant changes in ESR are not related to the structural orientation of cellular elements and molecules in the blood, but rather are determined by intramolecular rearrangements.

Unidirectional changes of ESR in both Rh-positive and Rh-negative blood also indicate a possible combined remote-field effect of the expressed subjective state of a person on various molecular structures in the blood, which we have already mentioned above. We can speculate that after mixing the blood, an effect of one of the factors associated with Rh-positive blood on ESR disappears, while there is still an effect of another factor on this parameter.

Everything, caused by expressed subjective remote influence of the investigator, changes of ESR remain within normal values, characteristic for the healthy person. When in experiences blood of sick people with high value of ESR (30 - $40 \mathrm{~mm}$ ) was taken, subjective exposure on blood didn't cause any changes of ESR.

\section{Conclusions}

The results of the present investigations clearly demonstrate the existence of the remote-field effect of the subjective state of a person. We called this brain field a "psychogenic field", because it reflects mental, subjective state of a person [13]. In our opinion [10] [11], neuronal brain structures are the "generator" of the psychogenic field and at the same time a "screen" that is affected by this field. It characterizes the closed cycle of the per- 
son's self-perception of the processes occurring in neuronal structures of the brain.

The nature of any field is determined by its action on the matter and by its influence on the processes. This is a common feature of all the known physical fields. There is no other way to detect the field and to describe its nature. All known field effects occur with maximum probability, which is reflected in our experiments.

It is now known that the psychogenic field of the brain causes the force acting on arrows of indicators, provides remote influence on physical properties of the blood, and can be detected only by means of living structures [10] [11]. It is further possible that there are still some other, yet unknown to us, manifestations of this field.

The data obtained provide opportunities for further search and isolation of molecular structures of the blood, which mediate its remote response to expressed subjective state of a person. We do not know yet, where the molecular structures of the blood, which are responsive to expressed subjective state of a person, are located in erythrocytes or in plasma. We hypothesize that these molecules may be proteins that change their conformational properties under the influence of expressed subjective states [10] [11].

\section{References}

[1] Sechenov, I.M. (1863) Brain Reflexes. Journal Medical Bulletin, 47, 461-484 and 48, 493-512. (In Russian)

[2] Pavlov, I.P. (1951) The Twenty Years of Objective Study of Animal Higher Nervous Activity (Behavior). In: Pavlov, I.P., Ed., Complete Compilation of Works, Vol. 3, The Publishing House of the Academy of Sciences of the USSR, Leningrad, 439 p. (In Russian)

[3] Sperry, R.W. (1952) Neurology and the Mind-Brain Problem. The Journal of American Science, 40, 291-312.

[4] Crick, F. and Koch, C. (1995) Why Neuroscience May Be Able to Explain Consciousness. Scientific American, 273, 84-85.

[5] Anokhin, P.K. (1969) Mental Form of Reflection of Reality. In: Pavlov, T., Ed., Lenin's Theory of Reflection, Part 1, Chapter 3, Science and Art, Sofia, 109. (In Russian)

[6] Bekhtereva, N.P. (1990) Per aspera... life. Science about a Brain of the Human. Science, Leningrad. (In Russian)

[7] Ivanitsky, A.M. (1999) The Main Secret of Nature: How Brain Processes Result in Subjective Experience. Psychological Journal, 20, 93-104. (In Russian)

[8] Nagel, T. (2001) Think Ability of Impossible and Problem of a Spirit and a Body. Philosophy Questions, 10, 92-107. (In Russian)

[9] Popper, K. (1994) Knowledge and the Body-Mind Problem. In: Notturno, M.A., Ed., Defence of Interaction, Routledge, London and New York, 170 p. (In English)

[10] Yumatov, E.A. (2013) Brain Psychic Activity: «Clue» for Understanding. Bulletin of the International Academy of Sciences. Russian Section, 1, 35-45. (In Russian) http://www.heraldrsias.ru/online/2013/1/269/

[11] Yumatov, E.A. (2014) To Knowledge of the Origin of the Brain Mental Activity. World Journal of Neuroscience, 4, 170-182. http://dx.doi.org/10.4236/wjns.2014.42020

[12] Yumatov, E.A. (2010) The Direct Registration of a Human Subjective State. Bulletin of New Medical Technologies, 4, 187-192. (In Russian)

[13] Yumatov, E.A. (2011) System Psychophysiology of Human Subjective State. Sputnik + Moscow. (In Russian)

[14] Yumatov, E.A. (2011) The Physics of Human Brain Subjective State. Consciousness and Physical Reality, 10, 39-50. (In Russian)

[15] Yumatov, E.A., Bykova, E.V., Dzhafarov, R.N. and Tsarenko, O. I. (2013) The Proof Remotely-Field Implication of a Subjective State of a Brain of a Human. Consciousness and Physical Reality, 2, 33-38. (In Russian)

[16] Yumatov, E.A., Bykova, E.V. and Dzhafarov, R.N. (2013) Long-Distance Effects of Human Subjective Status on the Blood Physicochemical Characteristics. Bulletin of Experimental Biology and Medicine, 155, 527-530. http://dx.doi.org/10.1007/s10517-013-2193-2 